\title{
KYNREFREV - implementation of an X-ray reverberation model in XSPEC
}

\section{D. Caballero-Garcia ${ }^{a *}$, M. Dovčiak ${ }^{a}$, I. Papadakis ${ }^{b c}$, A. Epitropakis ${ }^{b}$, J. Svoboda $^{a}$, E. Kara ${ }^{d}$, V. Karas ${ }^{a}$}

a Astronomical Institute of the Academy of Sciences, Boční II 1401, CZ-14100 Praha 4,

Czech Republic, E-mail: garcia@asu.cas.cz

${ }^{b}$ Department of Physics and Institute of Theoretical and Computational Physics, University of Crete, GR-71003 Heraklion, Greece

${ }^{c}$ Foundation for Research and Technology - Hellas, IESL, Voutes, GR-7110 Heraklion, Greece

${ }^{d}$ Department of Astronomy, University of Maryland, College Park, MD 20742-2421, USA

\begin{abstract}
We present briefly the first results obtained by the application of the KYNREFREV-reverberation model, which is ready for its use in XSPEC. This model computes the time dependent reflection spectra of the disc as a response to a flash of primary power-law radiation from a point source corona located on the axis of the black-hole accretion disc. The assumptions of the model are: central Kerr black hole, surrounded by a Keplerian, geometrically thin, optically thick, ionised disc with the possibility of defining the radial density profile and a stationary hot point-like patch of plasma located on the system rotation axis and emitting isotropic power-law radiation (lamppost geometry). Full relativistic ray-tracing code in vacuum is used for photon paths from the corona to the disc and to the observer and from the disc to the observer. The ionisation of the disc is set for each radius according to the amount of the incident primary flux and the density of the accretion disc. In this paper we comment on some preliminary results obtained through the analysis of X-ray reverberation time-lags from 1H 0707-495 and IRAS 13224-3809.
\end{abstract}

Accretion Processes in Cosmic Sources - APCS2016 -

5-10 September 2016,

Saint Petersburg, Russia

${ }^{*}$ Speaker. 


\section{Introduction}

The X-ray emission from accreting black holes (BHs), in particular the narrow line Seyfert 1 (NLS1) Active Galactic Nuclei (AGN) reported in this work, is believed to come from the innermost part of the accretion disc. This has been seen to be highly variable $[1,2,3]$. The accretion disc reprocesses and "reflects" the inner radiation, so part of the X-ray photons emitted by the corona are reflected and vary in luminosity partially according to variations in the flux received from the latter. The emission at the energy bands predominantly composed of reflection from the accretion disc (i.e. the soft-excess at $0.3-1 \mathrm{keV}$ and the broadened $\mathrm{Fe} \mathrm{K}_{\alpha}$ line at $\approx 3-8 \mathrm{keV}$ ) is expected to lag behind correlated variations in energy bands dominated by the directly observed continuum emission (1-4 keV). The time delay corresponds to the additional light travel time from the coronal source to the reflecting accretion disc [4]. Some of these sources show a reflection-dominated spectrum and potentially have maximally spinning $B H s$. This fact can be understood if light bending is an important effect for them [5]. Their high-energy source (i.e. the jet or corona) is very close to the $\mathrm{BH}$ and the observed direct continuum (i.e. power-law) is very low. This would correspond to Regime I of [5], corresponding to a low height of the primary source ( $h \leq 2-4 r_{g}$, depending on the observer inclination), where strong light-bending suffered by the primary radiation dramatically reduces the observed power-law emission component at infinity and the total luminosity at infinity stays relatively constant. During Regime II the height of the primary source is higher ( $\mathrm{h} \approx 4-13 \mathrm{r}_{\mathrm{g}}$, depending on the observer inclination) and the total luminosity at infinity varies by a factor of four and mostly due to variations of the primary source.

The recent detection of time delays, as a function of the Fourier frequency, between the softband and the hard-band photons in Active Galactic Nuclei (AGN), can shed light on the X-ray emission mechanism and the geometry of these BH systems. Currently, the observed negative $\mathrm{X}$-ray time-lags (i.e. soft-band variations lagging the hard band variations observed at high frequencies) have triggered a great deal of scientific interest on interpreting their nature. The first tentative detection was in the AGN Ark 564 [6], where an origin in reflection from the accretion disc was proposed. The first statistically significant detection came from the AGN 1H 0707-495 [7]. More recent works $[8,9]$ then found that such X-ray time-delays are much more common than it was initially thought by the analysis of the X-ray data from several tens of AGN [10]. The opposite time-delayed behaviour, i.e. positive X-ray time-delays (hard-band variations lag the soft band variations observed at low frequencies), has been known for quite some time in both AGN (e.g. $[11,12,13])$ and X-ray binaries (e.g. $[14,15,16])$. Although positive time-lags are expected in the standard Comptonization process within the X-ray source [16], they can also be produced by diffusive propagation of perturbations in the accretion flow [17].

In this paper we briefly describe a generalized scheme and a code which can be used with the standard X-ray spectral fitting package, XSPEC [18]. This model takes into account a treatment of all the general relativistic effects for accretion discs and light bending effects in the disc-corona geometry. Time-lags versus frequency and energy can be produced and fitted by the user, either in XSPEC or using another suitable tool. Sec. 1.1 shows a brief description of the model, Sec. 2 provides some preliminary results obtained using the model, and in Sec. 3 we briefly discuss and compare them with other results obtained in the literature. 


\subsection{The model}

This model (KYNREFREV; [19]) computes the X-ray emission from an accretion disc that is illuminated from the primary power-law source located on the axis above the central $\mathrm{BH}$ with a "flash" (hereafter referred to as "flare"). All relativistic effects are taken into account for photon paths in vacuum from the corona to the disc and to the observer and from the disc to the observer. The transfer functions (see [20]) for these effects have been precalculated and stored in the FITS files that are used by the model to speed up the execution of the code. The reflection is taken from the REFLIONX ${ }^{1}$ FITS table files [21], which are integrated over the disc.

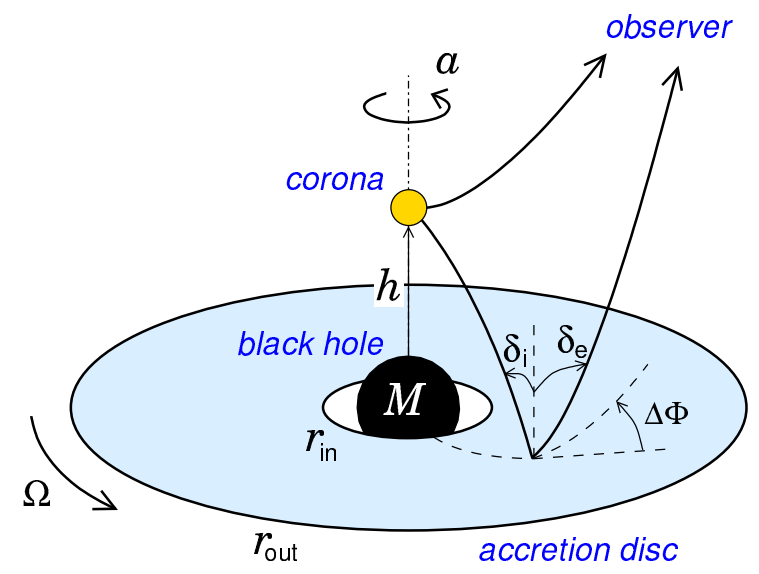

Figure 1: The sketch of the lamp-post geometry.

The lamp-post geometry used in the KYNREFREV model (see Fig. 1) consists of a BH, surrounded by an equatorial accretion disc, that is illuminated by an X-ray source located on the symmetry axis of the disc. Some of the main physical parameters of the model are the mass (in units of $\left.10^{8} \mathrm{M}_{\odot}\right)$ and the angular momentum $(-1 \leq \mathrm{a} / \mathrm{M} \leq 1)$, i.e. the spin of the $\mathrm{BH}$ in geometrized units, the height (h) of the X-ray source (in units of $\left.r_{g}=G M / c^{2}\right)$, and the viewing angle $\left(\theta_{0}\right)$ of a distant observer with respect to the axis of the disc.

The disc is assumed to be ionized, geometrically thin, Keplerian, co-rotating or counterrotating with the $\mathrm{BH}$, with a radial extent ranging from the inner edge of the disc (in units of the innermost stable circular orbit, $\left.\mathrm{r}_{\mathrm{ISCO}}\right)$ up to the outer radius $\left(\mathrm{r}_{\mathrm{out}}=10^{3} \mathrm{r}_{\mathrm{g}}\right)$. The spin of the BH uniquely defines $\mathrm{r}_{\mathrm{ISCO}}$. When measured in geometrized units, the spin can attain any value between zero and unity, with $a=0\left(\mathrm{r}_{\mathrm{ISCO}}=6 \mathrm{r}_{\mathrm{g}}\right)$ and $a=1\left(\mathrm{r}_{\mathrm{ISCO}}=1 \mathrm{r}_{\mathrm{g}}\right)$ indicating a non-spinning (i.e. Schwarzschild) and a maximally spinning (i.e. "extreme" Kerr) BH, respectively. The primary $\mathrm{X}$-ray source (i.e. corona) is assumed to be a hot cloudlet of plasma on the rotation axis at height $h$ and emitting a power-law spectrum of X-ray radiation, $\mathrm{F}_{\mathrm{p}} \propto \mathrm{E}^{-\Gamma^{\mathrm{E}} / \mathrm{E}_{\mathrm{c}}}$, with a sharp low-energy cut-off at $0.1 \mathrm{keV}$ and high-energy exponential cut-off at $\mathrm{E}_{\mathrm{c}}=300 \mathrm{keV}$.

In the new model the REFLIONX [21] tables for constant density slab illuminated by the power-law incident radiation are used to compute the local reprocessing of the incident radiation in the ionized accretion disc. The ionization of the disc, $\xi$, is set by the amount of the incident primary flux (dependent on the luminosity and height of the primary source and the mass of the $\mathrm{BH}$ ) and

\footnotetext{
${ }^{1}$ https://heasarc.gsfc.nasa.gov/xanadu/xspec/newmodels.html
} 
by the density of the accretion disc as $\xi(r) \propto \mathrm{F}(\mathrm{r}) / \mathrm{n}(\mathrm{r})$. We used a constant-density disc in our analysis. This simplification is well satisfied because the radial dependence of any realistic density profile is much less significant than the radial decrease of the disc illumination by the lamp-post corona (see, e.g., Fig. 3 in [22]).

We may consider several limb brightening/darkening prescriptions for the directionality of the re-processed emission: isotropic emission (flux $\sim 1$ ), Laor's limb darkening (flux $\sim(1+2.06 \mu$ ); [23]) and Haardt's limb brightening (flux $\ln (1+1 / \mu)$; [24, 25]), with $\mu$ being cosine of emission angle.

\subsubsection{Transfer function and time-lags}

We refer as reverberation to the variable signal that we get (i.e. hereafter called "response function") corresponding to the variations of the driving primary source of radiation [4]. We assume that the emission which we see in each band is related to the underlying driving signal by a linear impulse response, which represents the response of the emission in that band to an instantaneous "flash" (i.e. a delta-function impulse) in the underlying primary signal. Therefore, the observed light curve is the convolution of the underlying driving signal time-series with the impulse response for that energy band.

In this work we refer as "transfer function" the relative response of the disc to the illumination:

$$
\psi_{\mathrm{r}}(E, t)=\frac{\mathrm{F}_{\mathrm{r}}}{\mathrm{F}_{\mathrm{p}}}
$$

where $\mathrm{F}_{\mathrm{r}}(E, t)$ is the time dependent observed reflected flux from the disc as a response to a flare ${ }^{2}$ that would be observed as $\mathrm{F}_{\mathrm{p}} \delta(t)\left(\mathrm{F}_{\mathrm{p}}\right.$ is total observed primary flux at energy $\left.\mathrm{E}\right)$.

The Fourier transform of the transfer function is calculated as:

$$
\hat{\phi}_{\mathrm{r}}(E, f)=\mathrm{A}_{\mathrm{r}}(E, f) \mathrm{e}^{\phi_{\mathrm{r}}(E, f)}
$$

with amplitude $\mathrm{A}_{\mathrm{r}}(E, f)$ and phase $\phi_{\mathrm{r}}(E, f)$ (which is sometimes referred to as transfer function in other works).

And the total phase of the Fourier transform of the total flux (i.e. both the primary and the reflection continuum are taken into account) is:

$$
\phi_{\mathrm{tot}}(E, f)=\arctan \frac{\mathrm{A}_{\mathrm{r}}(E, f) \sin \phi_{\mathrm{r}}(E, f)}{1+\mathrm{A}_{\mathrm{r}}(E, f) \cos \phi_{\mathrm{r}}(E, f)} .
$$

One can calculate the time-lag of the signal, computed from the total phase at energy bin E with respect to the total phase at some reference energy bin:

$$
\tau(E, f)=\frac{\Delta \phi_{\mathrm{tot}}(E, f)}{2 \pi f}
$$

where $\Delta \phi_{\text {tot }}(E, f)$ is defined as the phase difference at any energy value $E$ with respect to the reference energy range $E_{\text {ref }}$, i.e. $\Delta \phi_{\text {tot }}(E, f)=\phi(E, f)-\phi\left(E_{\text {ref }}, f\right)$.

\footnotetext{
${ }^{2}$ Implicitly referred to a "Box" flare in practice because of numerical/computation reasons.
} 
To determine the response function of the disc, we assume that the primary X-ray source isotropically emits a flare of duration equal to $1 \mathrm{t}_{\mathrm{g}}\left(\mathrm{t}_{\mathrm{g}}=\mathrm{GM} / \mathrm{c}^{3}\right)$. However, after computing the response, the Fourier transform is corrected for the "box" shape of the primary flare so that it corresponds to the delta function impulse. Upon being illuminated, each area element of the disc "responds" to this flare by isotropically and instantaneously emitting a "reflection spectrum" in its rest-frame. We assume that the reprocessed flux is proportional to the incident flux, and that the disc material is ionized.

\section{Analysis and results}

\subsection{H 0707-495}

$1 \mathrm{H} \mathrm{0707-495} \mathrm{(z=0.0411)} \mathrm{is} \mathrm{a} \mathrm{narrow-line} \mathrm{Seyfert} 1$ galaxy with a BH mass estimate of $\mathrm{M}=$ $2 \times 10^{6} \mathrm{M}_{\odot}$ [26]. It is one of the most highly variable Seyfert galaxies, commonly known to increase by an order of magnitude in flux on timescales as short as one hour. Therefore this makes this source an ideal target for extensive spectral-timing analysis as previous studies based on the X-ray data from the XMM-Newton satellite have shown so far. [7] and [27] showed that the high-frequency variations in the soft excess from $0.3-1 \mathrm{keV}$ lag behind those in the $1-4 \mathrm{keV}$ band by $\sim 30 \mathrm{~s}$. They interpreted this short timescale lag as the reverberation time delay between the primary-emitting corona and the inner accretion disc. This short time delay would put the corona at a height of $<10 \mathrm{r}_{\mathrm{g}}$ from the accretion disc (using the aforementioned value for the $\mathrm{BH}$ mass).

\subsection{IRAS 13224-3809}

IRAS 13224-3809 ( $\mathrm{z}=0.066)$ is also one of the most $\mathrm{X}$-ray variable Seyfert 1 galaxies known [28], and therefore it is a useful source for which to probe the environments of the innermost regions around the BH. It was first observed with XMM-Newton in 2002 for $64 \mathrm{ks}$ [29, 30, 31], and later for $500 \mathrm{ks}$, which led to a significant detection of the soft time-lag [32]. Later [33] followed the source and reported on the analysis of the soft time-lags of IRAS 13224-3809 in the low and high flux states. Together with $1 \mathrm{H} \mathrm{0707-495}$ it is one of the very few sources for which XMM-Newton has devoted long-exposure ( $2500 \mathrm{ks}$ ) observational campaigns.

\subsection{Time-lag versus frequency spectral fitting}

We fitted the (0.3-1 versus 2-4 keV) time-lag versus frequency global spectrum of $1 \mathrm{H} 0707-$ 495 with the model KYNREFREV and obtained a very good fit $\left(\chi^{2} / v=28 / 30\right.$; with $v$ being the number of d.o.f.) as shown in Fig. 2. The primary isotropic flux was set to slightly sub-Eddington (i.e. $\mathrm{L} /$ Ledd $=0.1$ ) according to previous studies [7]. The value for the density of the accretion disc obtained was $\mathrm{N}_{\mathrm{H}}=(30 \pm 10) \times 10^{15} \mathrm{~cm}^{-3}$ (adopting a constant radial density profile) and the values of the rest of the parameters of the fit (spin, mass and height of the lamp-post) are shown in Tab. 1. The mass is in very good agreement with previous findings but the height of the lamp-post is higher $\left(5.8 \pm 0.5 r_{g}\right)$ than previously found $\left(2.4 \pm 0.5 r_{g}\right.$; [34]) and the value for the BH spin is lower than usually obtained from X-ray spectroscopy ( $>0.98$, e.g. [7]) but in agreement with the previous value obtained from time-lag fitting by [34] using a similar model. As shown in Fig. 3 the difference between the obtained slow-rotation (model A) and high-spinning BH (model B) is that 
in the latter we obtain phase wrapping at high frequencies (still not detected with current detectors). The extrapolation of the model with high spin to the lower (observed) frequencies is not compatible with the data, hence the reason of getting a lower value for the spin of the $\mathrm{BH}$.

When fitting the two datasets from IRAS 13224-3809 (the same as previously analyzed by [33]) we obtained also a good fit $\left(\chi^{2} / v=10 / 6\right)$ as shown in Fig. 4. We tested the "pure" lamppost geometry, in which the change of the state (i.e. luminosity) is due to the variable height of the corona. According to the expectation of a (near) Eddington accreting $\mathrm{BH}$ for the given mass [35] we set the primary isotropic flux to a slightly sub-Eddington value $(\mathrm{L} /$ Ledd $=0.1)$. The value for the density of the accretion disc obtained was $\mathrm{N}_{\mathrm{H}}=(26 \pm 5) \times 10^{15} \mathrm{~cm}^{-3}$ (adopting a constant radial density profile) and the values of the rest of the parameters of the fit (spin, mass and heights of the lamp-post during both states) are shown in Tab. 2. We obtain a moderate value for the spin $(0.74 \pm 0.02)$ that is lower than previously obtained $(a=0.99 ;[36])$

The reason for such a disagreement for the spin is the fact that for such a high value $(\gtrsim 0.95)$ phase-wrapping occurs (and it is more pronounced for high values of the intrinsic primary luminosity, after performing some testing). This can be seen in Fig. 5 where the current fitted model (model C) and a high-spinning BH (model D) are shown. If the spin is changed to a lower value (e.g. $\leq 0.5)$ phase-wrapping totally disappears.

As pointed previously [33] the change in the shape of the observed time-lags can be interpreted as a change in the properties of the corona. In the terms of the model and parametrization used this is shown in the form of a change in the height of the lamp-post (from $4.9 \pm 0.2 r_{g}$ to $9.0 \pm 1.0 r_{g}$ ). Both values are higher to what has been previously reported in the framework of the lamp-post model $\left(2.9 \pm 0.8 \mathrm{r}_{\mathrm{g}}\right.$; [34]), alike in the case of $1 \mathrm{H} 0707-495$, which might be due to the slightly different model used. Nevertheless, the values obtained for the Eddington ratio and coronal height are consistent with what has been obtained in recent work (see Fig. 7 by [10]).

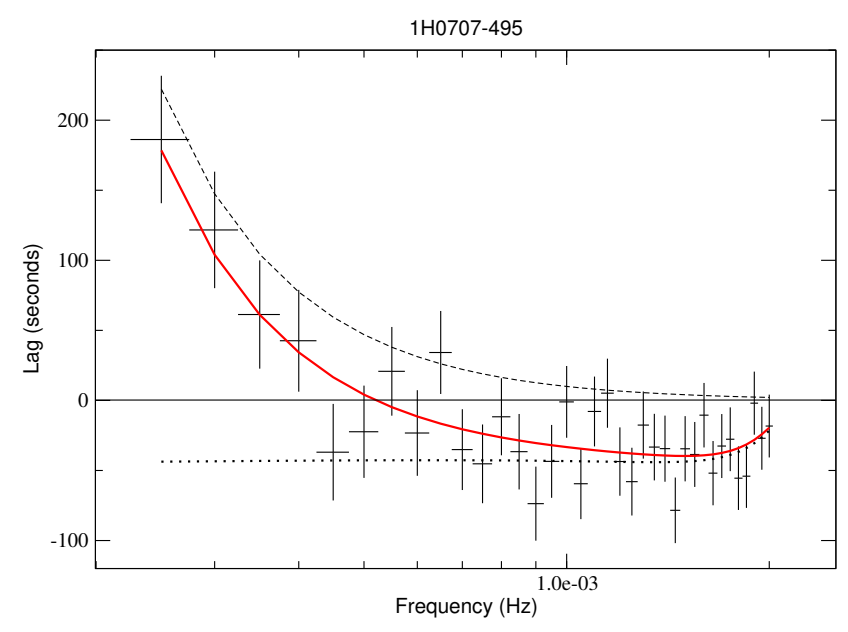

Figure 2: The (0.3-1 versus 2-4 keV) X-ray soft time-lag versus frequency spectrum of 1H 0707-495 fitted with the KYNREFREV model in XSPEC (red line). The two components of the best-fitting time-lag model are the relativistic reflected component (black-dotted line) and the power-law (black-dashed line). 

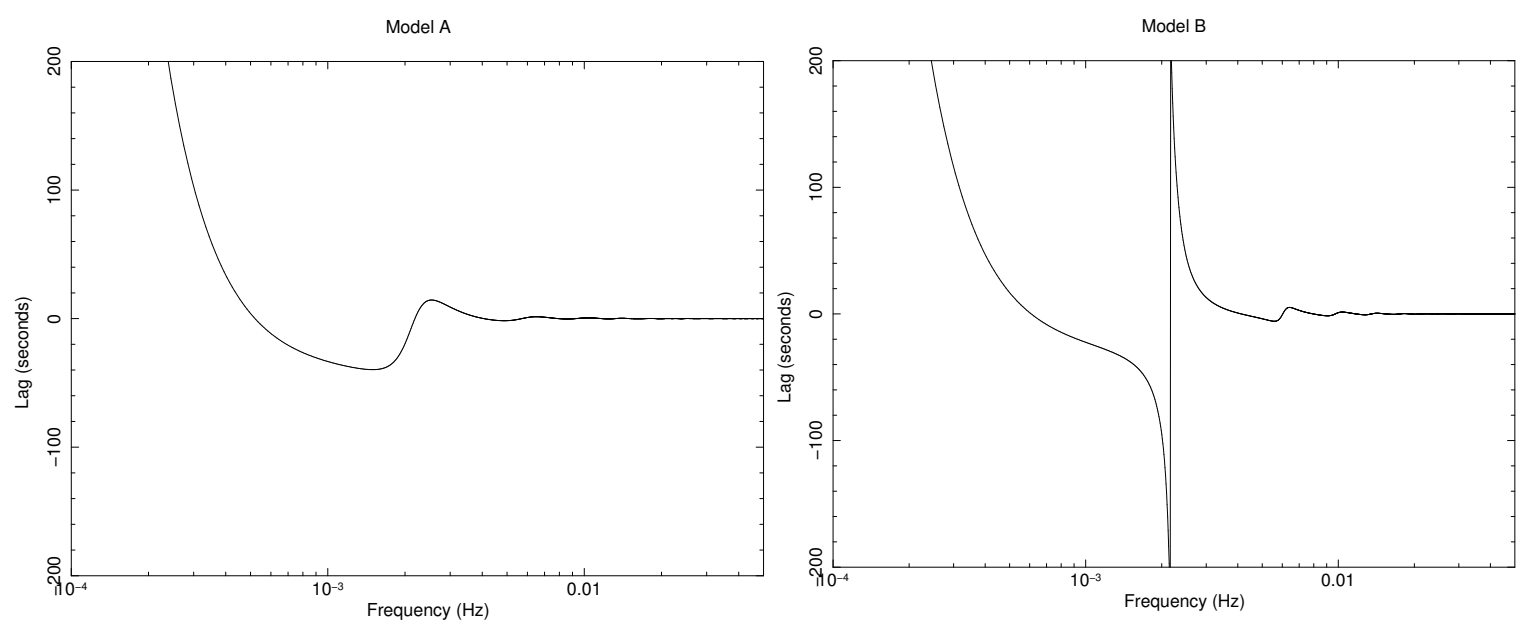

Figure 3: Extrapolated to higher frequencies fitted models for $1 \mathrm{H} 0707-495$ with the obtained value for spin given the data $(0.52 \pm 0.09$; model $\mathrm{A})$ and for a highly spinning $\mathrm{BH}(0.95$, model $\mathrm{B})$ at left and right, respectively.

\begin{tabular}{ccccccc}
\hline Mass & Spin & View. angle & Height & PL norm. & PL index & $\chi^{2} /$ d.o.f. \\
$\mathrm{M}$ & $\mathrm{a} / \mathrm{M}$ & $\theta_{\mathrm{o}}$ & $h$ & $\mathrm{~A}(\mathrm{~s})$ & $\mathrm{s}$ & \\
$\left(\times 10^{6} \mathrm{M}_{\odot}\right)$ & $(\mathrm{GM} / \mathrm{c})$ & $(\mathrm{deg})$. & $\left(\mathrm{GM} / \mathrm{c}^{2}\right)$ & & & \\
\hline $2.6 \pm 0.2$ & $0.52 \pm 0.09$ & $77 \pm 3$ & $5.8 \pm 0.5$ & $(2.2 \pm 0.3) \times 10^{-6}$ & $2.23 \pm 0.02$ & $28 / 30$ \\
\hline
\end{tabular}

Table 1: Values of the main parameters obtained from the best-fit using the KYNREFREV MODEL of the time-lags of 1H 0707-495 shown in Fig. 2. Errors are $68 \%$ confidence errors.

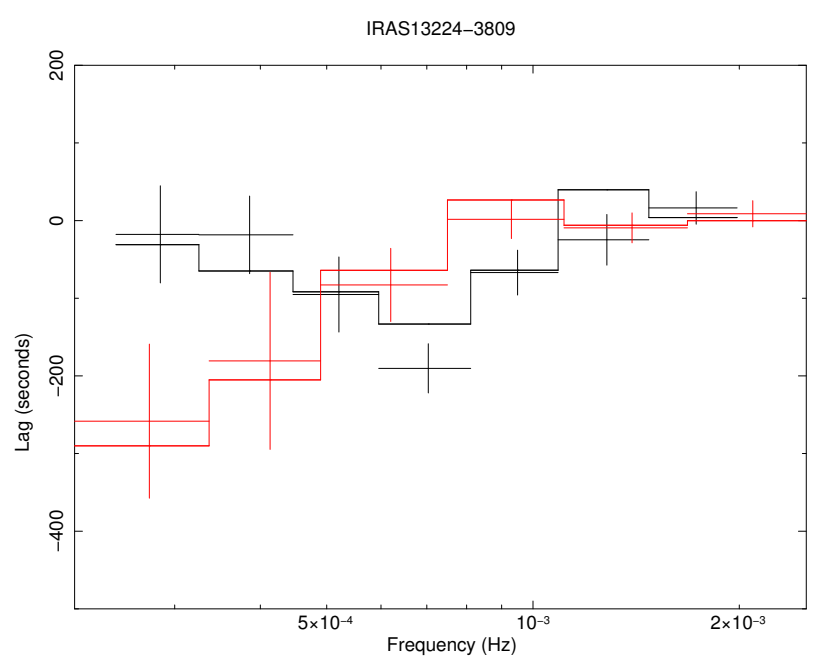

Figure 4: The (0.3-1 versus 1.2-5 keV) X-ray soft time-lag versus frequency spectrum of IRAS 132243809 fitted with the KYNREFREV model in XSPEC (black and red line, for the low and the high-flux states, respectively). 

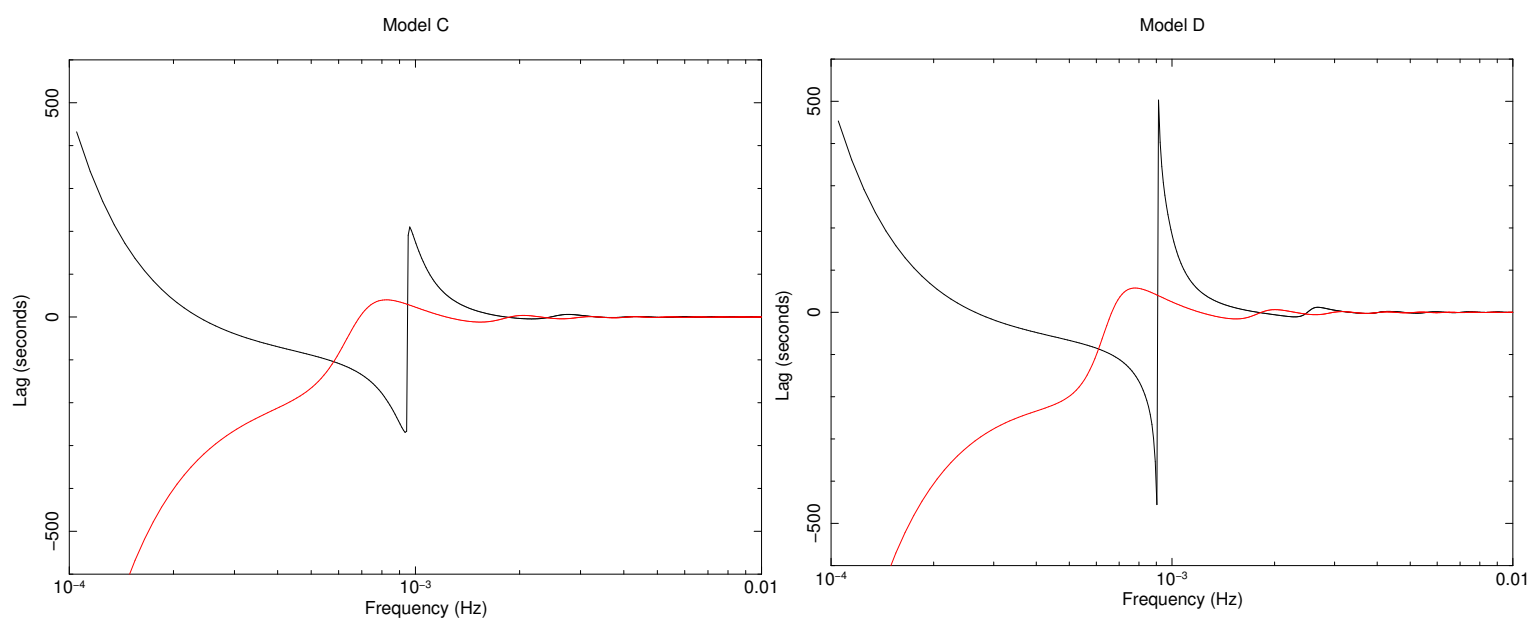

Figure 5: Extrapolated to higher frequencies fitted models for IRAS 13224-3809 with the obtained value for spin given the data $(0.74 \pm 0.02$; model $\mathrm{C})$ and for a highly spinning $\mathrm{BH}(0.95$, model $\mathrm{D})$ at left and right, respectively.

\begin{tabular}{cccccc}
\hline Mass & Spin & View. angle & Height (1) & Height (2) & $\chi^{2} /$ d.o.f. \\
$\mathrm{M}\left(\times 10^{6} \mathrm{M}_{\odot}\right)$ & $\mathrm{a} / \mathrm{M}(\mathrm{GM} / \mathrm{c})$ & $\theta_{\mathrm{o}}$ (deg.) & $h_{1}\left(\mathrm{GM} / \mathrm{c}^{2}\right)$ & $h_{2}\left(\mathrm{GM} / \mathrm{c}^{2}\right)$ & \\
\hline $7.1 \pm 0.2$ & $0.74 \pm 0.02$ & $66 \pm 5$ & $4.9 \pm 0.2$ & $9.0 \pm 1.0$ & $10 / 6$ \\
\hline
\end{tabular}

Table 2: Values of the main parameters obtained from the best-fit using the KYNREFREV MODEL of the time-lags of IRAS 13224-3809 shown in Fig. 4. Errors are $68 \%$ confidence errors.

\section{Discussion and conclusions}

In this paper we briefly presented a model (KYNREFREV) which deals with X-ray reverberation effects in AGN and can be used in and/or outside XSPEC. This model takes into account all the relativistic effects acting on light in the vicinity of the $\mathrm{BH}$ and a realistic prescription of the accretion disc surrounding it under the lamp-post primary source geometry. A more detailed description of the model will be presented in a forthcoming publication [19].

We present some preliminary interesting results obtained through the analysis of X-ray reverberation time-lags from 1H 0707-495 and IRAS 13224-3809 using this model. The global (0.3-1 versus $\approx 2-4 \mathrm{keV}$ ) X-ray soft time-lag spectrum is well fitted with this model, confirming previous statements on the X-ray reverberation origin of the negative X-ray soft time-lags [7]. We find that the BH is low and moderately-spinning for the case of 1H 0707-495 and IRAS 13224-3809, respectively. This is in contrast with previous results obtained from X-ray spectroscopy which suggest a maximally-spinning BH for both sources. We chose a near to Eddington value for the primary isotropic flux in the $2-10 \mathrm{keV}$ energy range of $\mathrm{L} / \mathrm{L} E D D=0.1$, which is reasonable given the masses of both BHs. We notice that these results are sensitive to the chosen $\mathrm{L} / \mathrm{L}_{\mathrm{EDD}}$ value, mostly in the case of IRAS 13224-3809, with a higher BH mass and hence with (observable with current instrumentation) phase-wrapping. We notice that, although our results are preliminary they are in agreement with previous claims of a low-spinning BH for the case of 1H 0707-495 [37].

In the case of IRAS $13224-3809$ we point out that the (0.3-1 vs $1.2-5 \mathrm{keV})$ spectral timelag transition related with the flux state of the source reported previously [33] might be due to a 
change in the height of the corona (from $4.9 \pm 0.2$ to $9.0 \pm 1.0 \mathrm{r}_{\mathrm{g}}$ in the low and the high-flux states, respectively). These results are consistent with the light-bending scenario proposed by [5], where in low-flux states the corona is closer to the $\mathrm{BH}$ and general relativistic effects are more important. If confirmed (with higher time-resolution observations), these results would indicate the power of analyzing X-ray reverberation time-lags in order to set further constraints of the inner disc-corona geometry for these systems.

\section{Acknowledgments}

MCG and MD acknowledge support provided by the European Seventh Frame-work Programme (FP7/2007-2013) under grant agreement $\mathrm{n}^{\circ}$ 312789. JS acknowledges financial support from the Grant Agency of the Czech Republic within the project n ${ }^{\circ} 14-20970 \mathrm{P}$.

\section{References}

[1] Leighly, K. M. ApJS, 125, 297, (1999)

[2] Turner, T. J., George, I. M., Nandra, K. \& Turcan, D. ApJ, 524, 667, (1999)

[3] Ponti, G., Papadakis, I., Bianchi, S., et al. $A \& A, \mathbf{5 4 2}$, 83, (2012)

[4] Uttley, P., Cackett, E. M., Fabian, A. C., et al. A\&ARv, 22, 72, (2014)

[5] Miniutti, G. \& Fabian, A. C. MNRAS, 349, 1435, (2004)

[6] McHardy, I. M., Arévalo, P., Uttley, P., et al. MNRAS, 382, 985, (2007)

[7] Fabian, A. C., Zoghbi, A., Ross, R. R., et al. Nature, 459, 540, (2009)

[8] Emmanoulopoulos, D., McHardy, I. M. \& Papadakis, I. E. MNRAS, 416, 94, (2011)

[9] De Marco, B., Ponti, G., Cappi, M., et al. MNRAS, 431, 2441, (2013)

[10] Kara, E., Alston, W. N., Fabian, A. C., et al. MNRAS, 462, 511, (2016)

[11] Papadakis, I. E., Nandra, K. \& Kazanas, D. ApJL, 554, 133, (2001)

[12] McHardy, I. M., Papadakis, I. E., Uttley, P., et al. MNRAS, 348, 783, (2004)

[13] Arévalo, P., Papadakis, I. E., Uttley, P. et al. MNRAS, 372, 401, (2006)

[14] Miyamoto, S. \& Kitamoto, S. Nature, 342, 773, (1989)

[15] Nowak, S. \& Vaughan, S. MNRAS, 280, 227, (1996)

[16] Nowak, M. A., Wilms, J. \& Dove, J. B. MNRAS, 517, 355, (1999)

[17] Kotov, O., Churazov, E. \& Gilfanov, M. MNRAS, 327, 799, (2001)

[18] Arnaud, K. A. Astronomical Society of the Pacific Conference Series, 101, 17, (1996)

[19] Dovčiak, M., Caballero-García, M. D., Papadakis, I. E., et al. In preparation, , , (2017)

[20] Dovčiak, M., Karas, V. \& Yaqoob, T. ApJS, 153, 205, (2004)

[21] Ross, R. R. \& Fabian, A. C., MNRAS, 358, 211, (2005)

[22] Svoboda, J., Dovčiak, M., Goosmann, R. W., et al. A\&A, 545, 106, (2012) 
[23] Laor, A. ApJ, 376, 90, (1991)

[24] Haardt, F. \& Matt, G. MNRAS, 261, 346, (1993)

[25] Czerny, B., Różańska, A., Dovčiak, M., et al. A\&A, 420, 1, (2004)

[26] Bian, W. \& Zhao, Y. MNRAS, 343, 164, (2003)

[27] Zoghbi, A., Fabian, A. C., Uttley, P., et al. MNRAS, 401, 2419, (2010)

[28] Boller, T., Brandt, W. N. \& Fink, H. A\&A, 305, 53, (1996)

[29] Boller, T., Tanaka, Y., Fabian, A., et al. MNRAS, 343, L89, (2003)

[30] Gallo, L. C., Boller, T., Tanaka, Y., et al. MNRAS, 347, 269, (2004)

[31] Ponti, G., Gallo, L. C., Fabian, A. C., et al. MNRAS, 406, 2591, (2010)

[32] Fabian, A. C., Zoghbi, A., Wilkins, D., et al. MNRAS, 419, 116, (2012)

[33] Kara, E., Fabian, A. C., Cackett, E. M., et al. MNRAS, 430, 1408, (2013)

[34] Emmanoulopoulos, D., Papadakis, I. E., Dovčiak, M. \& McHardy, I. M. MNRAS, 439, 3931, (2014)

[35] Boller, T., Brandt, W. N., Fabian, A. C. \& Fink, H. H. MNRAS, 289, 393, (1997)

[36] Fabian, A. C., Kara, E., Walton, D. J., et al. MNRAS, 429, 2917, (2012)

[37] Done, C. \& Jin, C. MNRAS, 460, 1716, (2016) 